\title{
"Winterização" de óleo de pescado via solvente
}

\author{
Winterization of fish oil with solvent \\ Daniele Colembergue CUNHA ${ }^{1}$, Valéria Texeira CREXI ${ }^{1}$, Luiz Antonio de Almeida PINTO ${ }^{1 *}$
}

\section{Resumo}

O fracionamento de óleos via "winterização" consiste numa cristalização fracionada, na qual os triacilgliceróis de óleos e gorduras são separados pela cristalização parcial na fase líquida. O objetivo deste trabalho consistiu na determinação das variáveis significativas e das condições operacionais do processo de "winterização" via solvente do óleo de pescado. Como matéria-prima empregou-se o óleo de pescado bruto de indústrias pesqueiras, o qual foi branqueado. Na etapa de "winterização", o óleo branqueado foi resfriado progressivamente em três estágios, de $30^{\circ} \mathrm{C}$ até $-5^{\circ} \mathrm{C}$. Foram estudados os seguintes fatores: tipo de solvente, proporção de solvente e agitação no segundo estágio do resfriamento. As composições de ácidos graxos dos óleos de pescado foram determinadas através de cromatografia gasosa. Foram analisadas as variações percentuais dos ácidos graxos insaturados e dos ácidos graxos saturados. As condições recomendadas para o processo foram o emprego de hexano como solvente, na proporção de $40 \%$ em relação à massa de óleo, e sem o emprego de agitação no segundo estágio do resfriamento. Sob tais condições, obteve-se um óleo com teor de ácidos graxos poli-insaturados (PUFA) de 64,3\%, sendo observado um aumento no percentual dos ácidos graxos insaturados de aproximadamente 9,2\% e uma redução de 13,4\% dos saturados, em relação ao óleo branqueado de pescado.

Palavras-chave: ácidos graxos; cristalização; fracionamento.

\begin{abstract}
The process of winterization consists of fractional crystallization, in which the triglycerides of oils and fats are separate by the partial crystallization in the liquid phase. The objective of this work was to evaluate significant variables and the operational conditions in the solvent winterization process of the fish oil. The crude fish oil from fishery industries was bleached and used as raw material. During the winterization step, the bleached oil was cooled progressively from $30^{\circ} \mathrm{C}$ to $5{ }^{\circ} \mathrm{C}$ in three phases. The solvent type, solvent proportion, and agitation in the second cooling stage were studied. The fatty acid compositions were determined through gas chromatography. Percentage variations of the contents of unsaturated and saturated fatty acids were analyzed. As a result, the use of hexane as solvent, $40 \%$ in relation to the oil mass without agitation in the second step of the cooling were recommended. Under such conditions, it were obtained an oil with polyunsaturated fatty acid content (PUFA) of $64.3 \%$. A percentage increase of approximately $9.2 \%$ in the content of unsaturated fatty acids and a percentage reduction of $13.3 \%$ in the content of saturated fatty acids in relation to bleached fish oil was observed.

Keywords: crystallization; fatty acids; fractionation.
\end{abstract}

\section{Introdução}

O óleo de pescado constitui uma importante fonte de ácidos graxos poli-insaturados ômega-3 (PUFA), principalmente ácidos eicosapentaenoico (EPA) e docosahexaenoico (DHA), esses ácidos graxos são benéficos à saúde humana, pois são essenciais para o desenvolvimento e funcionalidade de certos órgãos e para algumas respostas bioquímicas e fisiológicas do organismo (NAVARRO-GARCÍA et al., 2004; LIU et al., 2006).

O óleo bruto de pescado apresenta impurezas, necessitando assim passar por um processo de refino. $\mathrm{O}$ refino químico mostra-se mais adequado, pois o óleo bruto de pescado apresenta altos teores de fosfolipídios. Também existe a necessidade da introdução do fracionamento após o branqueamento para a obtenção de óleo rico em ômega-3 (MORAIS et al., 2001; CUNHA et al., 2002).

O refino do óleo a ser fracionado é essencial para o fracionamento, pois, segundo Rittner (1996), a natureza do óleo, os ácidos graxos livres, os mono e diacilgliceróis, as impurezas, os componentes menores e os inibidores de cristalização, assim como o polimorfismo de cristais formados, a intersolubilidade e a formação de cristais mistos, são fatores a serem considerados na realização de um processo de fracionamento.

Devido à importância nutricional e farmacêutica dos ácidos graxos poli-insaturados, cresce o interesse no desenvolvimento de processos que visam concentrar esses constituintes no óleo de pescado (NAVARRO-GARCÍA et al., 2004; COZZOLINO et al., 2005). Entre os procedimentos para o enriquecimento dos ácidos graxos insaturados no óleo, está a "winterização", que é um método físico de fracionamento que busca separar os triacilgliceróis de elevado ponto de fusão (causadores da turvação). Este método está baseado na precipitação dos triacilgliceróis na forma de cristais e filtração, sob determinadas condições de temperatura, tempo e agitação (THOMAS III, 1985;

Recebido para publicação em 7/9/2007

Aceito para publicação em 4/1/2009 (002835)

${ }^{1}$ Laboratório de Operações Unitárias, Escola de Química e Alimentos, Universidade Federal do Rio Grande - FURG, CP 474, CEP 96201-900, Rio Grande - RS, Brasil, E-mail: dqmpinto@furg.br

${ }^{*}$ A quem a correspondência deve ser enviada 
BERNARDINI, 1986; MASTROBATTISTA; GABRIELE, 1992; OCKERMAN; HANSEN, 1994; LÓPEZ-MARTÍNEZ et al., 2004). A cristalização pode ser dividida em três estágios básicos: super-resfriamento, nucleação e crescimento dos cristais. A distribuição de tamanho do cristal e forma é determinada pela maneira que o óleo é refrigerado e agitado (THOMAS III, 1985; OCKERMAN; HANSEN, 1994).

Existem três tipos básicos de fracionamento por "winterização": via seca, via solvente e via detergente (THOMAS III, 1985; HOFFMANN, 1989; SIMÕES, GIOIELLI, 1999). Thomas III (1985) e Bernardini (1986) conferem à "winterização" via solvente maior eficiência e melhores rendimentos em relação ao fracionamento realizado via seca, além de redução no tempo de operação e aumento da pureza. Este método é particularmente importante para triacilgliceróis formados de ácidos graxos de cadeia longa, que tendem a formar um líquido viscoso e/ou sólido acima da faixa de temperatura desejável para fracionamento.

A escolha do solvente depende de fatores como o tipo de triacilgliceróis presentes no óleo e as características desejadas para o produto fracionado. Porém outros fatores também devem ser considerados, como estabilidade, perdas voláteis, requerimento de energia para resfriar e para completa remoção do solvente (OCKERMAN; HANSEN, 1994). O hexano é o solvente mais comumente utilizado no fracionamento comercial de gorduras, porém outros solventes, como acetona, álcool isopropílico, isobutanol e etanol, também são citados como habituais (THOMAS III, 1985; BERNARDINI, 1986; TRUJILLO-QUIJANO et al., 1988; MASTROBATTISTA; GABRIELE, 1992; LÓPEZ-MARTÍNEZ et al., 2004).

Este trabalho tem como objetivo definir as variáveis significativas na "winterização" via solvente do óleo de pescado, e determinar as condições operacionais adequadas desta etapa no processo de refino para o enriquecimento do óleo em ácidos graxos poli-insaturados.

\section{Material e métodos}

\subsection{Matéria-prima}

O óleo bruto de pescado utilizado nos experimentos de "winterização" foi cedido por uma indústria local processadora de farinha de pescado, a qual processa a farinha através da cocção e posterior prensagem dos rejeitos de pescado, obtendo o óleo bruto. Os rejeitos da planta de fileteamento consistem basicamente da cabeça, espinhaço e vísceras de diversas espécies de pescado, principalmente castanha, corvina e pescada. As amostras do óleo bruto foram armazenadas, até serem utilizadas, em recipientes escuros, ao abrigo da luz e sob refrigeração.

\subsection{Refino do óleo bruto de pescado}

Para obtenção do óleo branqueado, realizou-se o processamento do óleo bruto de pescado segundo metodologia proposta por Morais et al. (2001), que consta das seguintes etapas: degomagem, neutralização, lavagem, secagem e branqueamento. Os experimentos para obtenção do óleo branqueado foram realizados em duplicata.
No processo de refino, a etapa de degomagem foi realizada durante $30 \mathrm{~min}$ a uma temperatura de $80^{\circ} \mathrm{C}$ e agitação de $500 \mathrm{rpm}$, com adição de $1,0 \%$ de ácido fosfórico $(85 \%$ p/p) em relação à massa do óleo. A etapa de neutralização ocorreu durante 20 minutos, com uma temperatura de $40^{\circ} \mathrm{C}$ e agitação de $500 \mathrm{rpm}$, com adição de solução de hidróxido de sódio $20 \%$ p/p (utilizando-se 4,0\% de excesso em relação ao índice de acidez determinado após a etapa da degomagem). Após cada etapa, o material foi centrifugado por 20 minutos a 7000 x g (Centrífuga Modelo Sigma 6-15, D-37520 Osterode, Alemanha), para a separação do óleo.

$\mathrm{Na}$ etapa de lavagem, ocorreu a adição de 10\% de água em relação à massa de óleo a $95^{\circ} \mathrm{C}$ durante um tempo de contato de 10 minutos, com agitação de $500 \mathrm{rpm}$ e temperatura do óleo mantida a $50{ }^{\circ} \mathrm{C}$. Essa etapa foi repetida três vezes. A etapa de secagem teve a duração de 20 minutos com uma temperatura de 90 a $95^{\circ} \mathrm{C}$ e agitação de $500 \mathrm{rpm}$. A etapa de branqueamento foi realizada à temperatura de $70^{\circ} \mathrm{C}$, agitação de $40 \mathrm{rpm}$, com adição de 5\% de adsorvente (mistura de terra ativada Fulmont Première e carvão ativado na relação de 9:1), sendo o tempo de contato de 20 minutos. A filtração foi realizada em funil de Büchnner com uma pré-capa de terra diatomácea. Todas as etapas do refino foram realizadas com pressão manométrica de vácuo de aproximadamente $700 \mathrm{mmHg}$.

\subsection{Fracionamento do óleo de pescado}

O óleo branqueado de pescado foi fracionado via "winterização", conforme mostra o diagrama de blocos da Figura 1.

Para cada experimento, utilizou-se uma quantidade de $200 \mathrm{~mL}$ de óleo branqueado, no qual se realizou a "winterização" via solvente, empregando a curva de resfriamento citada por Bernardini (1985). As condições operacionais dessa curva encontram-se apresentadas na Tabela 1. O processo ocorreu, no primeiro estágio, em estufa incubadora, até $5^{\circ} \mathrm{C}$, e no segundo

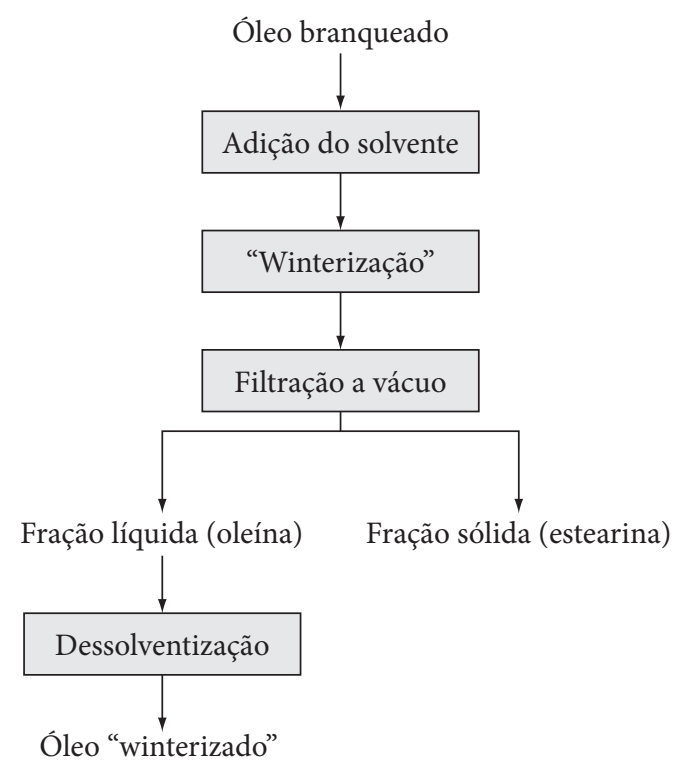

Figura 1. Diagrama de blocos do processo de fracionamento via "winterização" com solvente do óleo branqueado de pescado. 
e terceiro estágios, em banho termostatizado utilizando mistura álcool e água, sendo cada estágio realizado conforme a taxa de resfriamento desejada, até atingir a temperatura de $-5^{\circ} \mathrm{C}$.

A filtração para separação das frações oleína e estearina foi realizada com vácuo de 200-300 $\mathrm{mmHg}$, utilizando, como meio filtrante, tecido de algodão com pré-capa de terra diatomácea. A dessolventização da fração oleína ocorreu em balão com agitação, à temperatura de aproximadamente $80^{\circ} \mathrm{C}$ e sob vácuo de $500 \mathrm{mmHg}$. Após a filtração do óleo "winterizado", obtiveram-se $120 \mathrm{~mL}$ da fração oleína, sendo este o rendimento desta fração de $60 \%$.

\subsection{Metodologia analítica}

Para a caracterização dos óleos bruto e branqueado, foram determinados os seguintes parâmetros: Ácidos Graxos Livres (AGL), Índice de Peróxidos (IP), Índice de Iodo (II) e Índice de Saponificação (IS), empregando as metodologias da American Oil Chemists Society, AOCS $(1980,2002)$.

Realizou-se cromatografia gasosa para identificação e quantificação dos ácidos graxos dos óleos clarificado e "winterizado”, em equipamento (Modelo CGC AGILEN 6850 séries GC SYSTEM, EUA) com coluna megabore de $0,53 \mathrm{~mm}$ de diâmetro externo e $30 \mathrm{~m}$ de comprimento.

No método gás cromatográfico, empregou-se nitrogênio como gás de arraste (fluxo de $30 \mathrm{~mL} \cdot \mathrm{min}^{-1}$ ), temperatura do injetor de $250^{\circ} \mathrm{C}$, temperatura do detector de $270{ }^{\circ} \mathrm{C}$ e temperatura inicial da coluna de $160^{\circ} \mathrm{C}$. A programação da temperatura da coluna foi $160{ }^{\circ} \mathrm{C}$ por 1 minuto, seguida de aumento a uma taxa de $2{ }^{\circ} \mathrm{C} /$ minuto até $200{ }^{\circ} \mathrm{C}$; permanecendo a $200{ }^{\circ} \mathrm{C}$ por 2 minutos. Em seguida, houve novo aumento a uma taxa de $20^{\circ} \mathrm{C}$ /minuto até $220^{\circ} \mathrm{C}$; permanecendo nesta temperatura por 40 minutos. Para injeção no equipamento, os ésteres metílicos foram preparados segundo a metodologia de Maia (1992). A quantidade de amostra injetada foi de $1 \mu \mathrm{L}$. Os ácidos graxos foram identificados pela comparação direta dos tempos de retenção com padrões (Sigma Supelco). A quantificação dos ácidos graxos foi determinada pelo método de normalização de área, através do software Varian Star 4.51.

\section{Metodologia estatística}

Para analisar os fatores significativos no processo de "winterização" com solvente e definir a condição adequada de trabalho, foi realizado um planejamento fatorial completo $2^{3}$, conforme apresentado em Box et al. (1978).

Tabela 1. Condições operacionais do processo da etapa de "winterização" no fracionamento do óleo de pescado.

\begin{tabular}{lccc}
\hline \multicolumn{1}{c}{ Condições } & \multicolumn{3}{c}{ Estágio } \\
\cline { 2 - 4 } & $1^{\circ}$ Estágio & $2^{\circ}$ Estágio & $3^{\circ}$ Estágio \\
\hline Temperatura inicial $\left({ }^{\circ} \mathrm{C}\right)$ & 30 & 5 & -4 \\
Taxa de resfriamento $\left({ }^{\circ} \mathrm{C} / \mathrm{h}\right)$ & 30 & 2,7 & 0,25 \\
Agitação $(\mathrm{rpm})$ & 600 & 0 ou $40^{*}$ & 0 \\
Temperatura final: & $5{ }^{\circ} \mathrm{C}$ & $-4{ }^{\circ} \mathrm{C}$ & $-5^{\circ} \mathrm{C}$ \\
\hline
\end{tabular}

${ }^{*}$ Conforme a matriz do planejamento experimental.
Os valores dos níveis dos fatores de estudos utilizados na “winterização" via solvente foram determinados através das referências bibliográficas Bernardini (1986) e Thomas III (1985). Sendo que, para o fator solvente $\left(\mathrm{S}_{\text {cod }}\right)$, o menor nível utilizado foi a acetona (nível -1) e o maior nível, o hexano (nível +1); para o fator proporção de solvente ( $\mathrm{PS}_{\text {cod }}$ ), o menor valor foi de $40 \%$ (nível -1) e o maior, de 60\% (nível +1); e, para o fator agitação no segundo estágio $\left(\mathrm{A}_{\text {cod }}\right)$, o menor valor foi de zero (nível -1) e o maior foi de $40 \mathrm{rpm}$ (nível +1).

A matriz do planejamento experimental, utilizada no processo de "winterização" de óleo branqueado de pescado, nas formas não-codificada e codificada, é apresentada na Tabela 2. Os ensaios experimentais da matriz do planejamento foram realizados em duplicata.

Através da análise de regressão, obteve-se o modelo teórico estatístico na forma codificada, considerando-se os efeitos principais e suas interações para as respostas aumento do percentual de ácidos graxos insaturados e redução do percentual de saturados, sendo este representado na Equação 1.

$Y_{i}=A+B X_{1}+C X_{2}+D X_{3}+E X_{1} X_{2}+F X_{1} X_{3}+G X_{2} X_{3}$

onde $A, B, C, D, E, F, G$, representam os parâmetros do modelo estatístico de primeira ordem; $X_{1}, X_{2}, X_{3}$, as variáveis de estudo na forma codificada (solvente, proporção de solvente e agitação no $2^{\circ}$ estágio, respectivamente); e $Y_{1}$, as respostas consideradas (aumento do percentual de ácidos graxos insaturados e redução do percentual de saturados) nos seus valores reais.

\section{Resultados e discussão}

\subsection{Caracterização da matéria-prima}

A Tabela 3 apresenta os valores para os índices de iodo (II), de saponificação (IS) e de peróxido (IP), e o percentual de ácidos graxos livres (\%AGL) para a matéria-prima (óleo bruto de pescado) e para o óleo branqueado utilizado nos experimentos de "winterização".

Os índices de Iodo e de Saponificação do óleo bruto de pescado apresentados na Tabela 3 estão dentro da faixa citada por Bernadini (1985) para diferentes espécies de animais marinhos

Tabela 2. Matriz do planejamento experimental fatorial completa $\left(2^{3}\right)$, utilizada no processo de "winterização" de óleo branqueado de pescado.

\begin{tabular}{cccc}
\hline Ensaio & $\begin{array}{c}\text { Solvente } \\
\text { tipo }\left(\mathrm{S}_{\text {cod }}\right)\end{array}$ & $\begin{array}{c}\text { Proporção de } \\
\text { solvente } \\
(\%)\left(\mathrm{PS}_{\text {cod }}\right)\end{array}$ & $\begin{array}{c}\text { Agitação no } \\
2^{\circ} \text { estágio } \\
(\mathrm{rpm})\left(\mathrm{A}_{\text {cod }}\right)\end{array}$ \\
\hline 1 & acetona -1 & $40-1$ & $0-1$ \\
2 & hexano +1 & $40-1$ & $0-1$ \\
3 & acetona -1 & $60+1$ & $0-1$ \\
4 & hexano +1 & $60+1$ & $0-1$ \\
5 & acetona -1 & $40-1$ & $40+1$ \\
6 & hexano +1 & $40-1$ & $40+1$ \\
7 & acetona -1 & $60+1$ & $40+1$ \\
8 & hexano +1 & $60+1$ & $40+1$ \\
\hline
\end{tabular}


(II de 110 a 195 cg I/g e IS de 160 a 196 mg KOH/g). Após o branqueamento do óleo de pescado, estes índices apresentaram pouca diferença em relação ao óleo bruto, ficando ambos dentro do erro da análise da determinação. Isto se deve ao fato do branqueamento do óleo bruto não afetar a composição de ácidos graxos dos triacilgliceróis, porém remover fosfolipídios, ácidos graxos livres, pigmentos e outras impurezas que afetam a estabilidade do produto de maneira adversa.

Verifica-se também, na Tabela 3, que os valores encontrados para o índice de peróxido e percentual de ácidos graxos livres foram inferiores no óleo branqueado em relação ao óleo bruto. Isto era esperado, pois as etapas do refino têm por objetivo melhorar as características do óleo de pescado, ou seja, retirar impurezas, ácidos graxos livres e produtos de oxidação lipídica.

A Tabela 4 apresenta as composições de ácidos graxos do óleo branqueado. Na Tabela 5, estão apresentadas as composições de ácidos graxos dos óleos "winterizados" conforme a matriz experimental apresentada na Tabela 2.

Analisando a Tabela 5, verifica-se, como esperado, que ocorreu redução na composição de ácidos graxos saturados e

Tabela 3. Caracterização dos óleos bruto e branqueado de pescado.

\begin{tabular}{lcc}
\hline \multicolumn{1}{c}{ Determinações } & Óleo bruto* $^{*}$ & Óleo clarificado* \\
\hline Índice iodo, II (cg I/g) & $133 \pm 2$ & $135 \pm 1$ \\
Índice saponificação, IS (mg KOH/g) & $185 \pm 2$ & $186 \pm 2$ \\
Índice peróxido, IP (meq O/ $/ \mathrm{kg})$ & $2,1 \pm 0,3$ & $1,7 \pm 0,2$ \\
$\%$ Ac. graxos livres (\% ác. oleico) & $1,60 \pm 0,03$ & $0,25 \pm 0,01$ \\
\hline *Valor médio \pm erro médio (em duplicata). & &
\end{tabular}

Tabela 4. Composições de ácidos graxos da amostra de óleo branqueado de pescado utilizadas nos experimentos de "winterização".

\begin{tabular}{cc}
\hline Ácido graxo & Óleo branqueado (\%) \\
\hline C14:0 $15: 0$ & $5,2 \pm 0,1$ \\
C16:0 & $0,7 \pm 0,1$ \\
C16:1 $19,6 \pm 0,1$ \\
C17:0 $18: 0$ & $9,5 \pm 0,1$ \\
C18:1 & $0,9 \pm 0,1$ \\
C18:2 & $4,9 \pm 0,1$ \\
C18:3 $22,3 \pm 0,1$ \\
C20:0 $20: 1$ & $2,1 \pm 0,1$ \\
C20:4 & $4,3 \pm 0,1$ \\
C20:5 & $0,5 \pm 0,1$ \\
C22:0 & $1,9 \pm 0,1$ \\
C22:1 & $0,8 \pm 0,1$ \\
C22:6 & $7,3 \pm 0,1$ \\
$\Sigma s^{* *}$ & $1,7 \pm 0,1$ \\
Sins & $0,5 \pm 0,1$ \\
$\Sigma$ n.i. & $10,2 \pm 0,2$ \\
\hline
\end{tabular}

${ }^{*}$ valor médio \pm erro médio (em duplicata); ${ }^{* *} \Sigma$ s: somatório saturados; $\Sigma$ ins: somatório insaturados; e $\Sigma$ n.i: somatório não identificados. aumento na composição de ácidos graxos insaturados e polinsaturados. Entretanto, essa variação não foi muito expressiva na composição de cada ácido graxo. Dessa maneira, realizou-se a análise das respostas em relação à variação percentual do total de ácidos graxos saturados e do total da variação percentual de ácidos graxos insaturados.

A partir das Tabelas 4 e 5, calcularam-se a variação no percentual de ácidos graxos insaturados e a variação no percentual dos ácidos graxos saturados. Estes percentuais foram calculados conforme apresentam as Equações 2 e 3.

$\%$ variação $\operatorname{\Sigma ins}=\frac{\left[\operatorname{\sum ins}(o w)-\operatorname{Sins}(o c)\right] * 100}{\operatorname{Sins}(o c)}$

onde:

- Lins (ow) é o percentual de insaturados no óleo winterizado;

- Lins (oc) é o percentual de insaturados no óleo branqueado.

$\%$ var iação $\Sigma s=\frac{[\Sigma s(o w)-\Sigma s(o c)] * 100}{\Sigma s(o c)}$

onde:

- $\Sigma$ s (ow) é o percentual de saturados no óleo winterizado;

- $\Sigma$ s (oc) é o percentual de saturados no óleo branqueado.

Os valores obtidos das Equações 2 e 3 são apresentados na Tabela 6.

A Tabela 7 apresenta a análise de variância das variações nos percentuais de ácidos graxos insaturados e de saturados, tendo como estudo o tipo de solvente (Scod), sua proporção (PScod) e a agitação no $2^{\circ}$ estágio do processo de "winterização" (Acod).

Observa-se, através da Tabela 7, para as duas respostas consideradas (aumento no percentual de ácidos graxos insaturados e redução no percentual de saturados), que, ao nível de significância de 95\% (valores de significância menores ou iguais a 0,05), a proporção de solvente (PScod) e a interação proporção: agitação no $2^{\circ}$ estágio (PscodAcod) do processo são significativas. Também se constata que a proporção de solvente (PScod) apresentou maior influência sobre a resposta, seguida, respectivamente, de suas interações com o tipo de solvente (Scod) e agitação no $2^{\circ}$ estágio (Acod) do processo para ambos os estudos. Verifica-se também, na Tabela 7, que, apesar do tipo de solvente (Scod) não se mostrar significativo ao nível de $95 \%$ (valor de significância menor ou igual a 0,05 ) para ambos os estudos, este, porém, permaneceu na análise, pois a sua interação com a agitação mostrou-se muito significativa, sendo a de maior influência sobre as respostas consideradas.

As Equações 4 e 5 representam o modelo teórico estatístico (Equação 1) para o aumento no percentual de ácidos graxos insaturados (\% Insat.) e a redução do percentual de saturados (\% Sat.).

$$
\begin{aligned}
& \% \text { Insat }=5,67+0,15 \mathrm{~S}_{\text {cod }}-1,3 \mathrm{PS}_{\text {cod }}-0,9 \mathrm{~A}_{\text {cod }}- \\
& -0,52 \mathrm{~S}_{\text {cod }} \mathrm{PS}_{\text {cod }}-1,57 \mathrm{~S}_{\text {cod }} \mathrm{A}_{\text {cod }}-0,97 \mathrm{PS}_{\text {cod }} \mathrm{A}_{\text {cod }}
\end{aligned}
$$


Tabela 5. Composições de ácidos graxos dos óleos de pescado "winterizados" conforme matriz experimental.

\begin{tabular}{|c|c|c|c|c|c|c|c|c|}
\hline Ácido graxo & Ensaio $1^{\star}(\%)$ & Ensaio $2^{*}(\%)$ & Ensaio $3^{*}(\%)$ & Ensaio $4^{*}(\%)$ & Ensaio $5^{\star}(\%)$ & Ensaio $6^{*}(\%)$ & Ensaio $7^{\star}(\%)$ & Ensaio $8^{*}(\%)$ \\
\hline C14:0 & $5,0 \pm 0,1$ & $4,8 \pm 0,1$ & $4,9 \pm 0,3$ & $4,6 \pm 0,1$ & $4,6 \pm 0,3$ & $5,0 \pm 0,4$ & $4,9 \pm 0,3$ & $5,0 \pm 0,3$ \\
\hline C15:0 & $0,6 \pm 0,1$ & $0,4 \pm 0,1$ & $0,7 \pm 0,1$ & $0,7 \pm 0,1$ & $0,5 \pm 0,1$ & $0,6 \pm 0,1$ & $0,7 \pm 0,1$ & $0,7 \pm 0,1$ \\
\hline $\mathrm{C} 16: 0$ & $18,3 \pm 0,2$ & $17,0 \pm 0,1$ & $18,3 \pm 0,2$ & $17,8 \pm 0,3$ & $17,7 \pm 0,4$ & $18,3 \pm 0,6$ & $18,3 \pm 0,7$ & $19,2 \pm 0,6$ \\
\hline C16:1 & $10,0 \pm 0,1$ & $10,2 \pm 0,1$ & $10,1 \pm 0,2$ & $10,0 \pm 0,1$ & $10,3 \pm 0,3$ & $10,0 \pm 0,3$ & $10,0 \pm 0,3$ & $9,5 \pm 0,3$ \\
\hline C17:0 & $0,9 \pm 0,1$ & $0,7 \pm 0,1$ & $0,8 \pm 0,1$ & $0,9 \pm 0,1$ & $0,7 \pm 0,1$ & $0,7 \pm 0,1$ & $0,9 \pm 0,1$ & $0,8 \pm 0,1$ \\
\hline C18:0 & $4,3 \pm 0,1$ & $4,0 \pm 0,4$ & $4,5 \pm 0,1$ & $4,2 \pm 0,1$ & $4,0 \pm 0,1$ & $4,0 \pm 0,1$ & $4,1 \pm 0,1$ & $4,9 \pm 0,1$ \\
\hline C18:3 & $4,5 \pm 0,3$ & $4,4 \pm 0,1$ & $4,3 \pm 0,1$ & $4,5 \pm 0,1$ & $4,4 \pm 0,1$ & $4,4 \pm 0,1$ & $4,3 \pm 0,5$ & $4,3 \pm 0,1$ \\
\hline C20:0 & $0,5 \pm 0,1$ & $0,5 \pm 0,1$ & $0,4 \pm 0,1$ & $0,5 \pm 0,1$ & $0,4 \pm 0,1$ & $0,5 \pm 0,1$ & $0,5 \pm 0,1$ & $0,5 \pm 0,1$ \\
\hline C20:1 & $2,0 \pm 0,1$ & $2,0 \pm 0,1$ & $1,9 \pm 0,1$ & $1,9 \pm 0,1$ & $2,1 \pm 0,1$ & $1,9 \pm 0,1$ & $1,8 \pm 0,1$ & $1,9 \pm 0,1$ \\
\hline C20:4 & $0,9 \pm 0,1$ & $1,3 \pm 0,1$ & $0,9 \pm 0,1$ & $1,0 \pm 0,1$ & $1,2 \pm 0,1$ & $0,8 \pm 0,1$ & $0,6 \pm 0,2$ & $0,9 \pm 0,1$ \\
\hline$\Sigma \mathrm{s}$ & $31,2 \pm 0,8$ & $29,0 \pm 1,0$ & $30,9 \pm 1,0$ & $29,7 \pm 0,9$ & $29,3 \pm 1,2$ & $30,1 \pm 1,5$ & $31,1 \pm 1,4$ & $32,7 \pm 1,4$ \\
\hline Sins & $61,6 \pm 1,2$ & $64,3 \pm 1,1$ & $61,9 \pm 1,3$ & $63,3 \pm 1,1$ & $63,6 \pm 1,0$ & $62,5 \pm 1,4$ & $61,5 \pm 2,4$ & $59,3 \pm 1,2$ \\
\hline$\Sigma$ n.i. & 7,2 & 6,7 & 7,2 & 7,0 & 7,1 & 7,4 & 7,4 & 8,0 \\
\hline
\end{tabular}

*valor médio \pm erro médio (em duplicata).

Tabela 6. Resultados da matriz experimental para as respostas consideradas.

\begin{tabular}{ccc}
\hline Ensaio & $\begin{array}{c}\text { Aumento no } \\
\text { percentual de insaturados }\end{array}$ & $\begin{array}{c}\text { Redução no } \\
\text { percentual de saturados }\end{array}$ \\
\hline 1 & $4,6 \pm 0,5$ & $6,9 \pm 0,5$ \\
2 & $9,2 \pm 0,3$ & $13,4 \pm 1,2$ \\
3 & $5,1 \pm 0,6$ & $7,8 \pm 1,1$ \\
4 & $7,4 \pm 0,2$ & $11,3 \pm 0,8$ \\
5 & $8,0 \pm 0,2$ & $12,5 \pm 1,7$ \\
6 & $6,1 \pm 0,7$ & $10,1 \pm 2,6$ \\
7 & $4,4 \pm 0,2$ & $7,2 \pm 1,2$ \\
8 & $0,6 \pm 0,1$ & $2,3 \pm 0,8$ \\
\hline
\end{tabular}

*valor médio \pm erro médio (em duplicata).

$$
\begin{aligned}
& \% \text { Sat }=8,93+0,33 \mathrm{~S}_{\text {cod }}-1,78 \mathrm{PS}_{\text {cod }}-0,91 \mathrm{~A}_{\text {cod }}- \\
& -0,68 \mathrm{~S}_{\text {cod }} \mathrm{PS}_{\text {cod }}-2,16 \mathrm{~S}_{\text {cod }} \mathrm{A}_{\text {cod }}-1,48 \mathrm{PS}_{\text {cod }} \mathrm{A}_{\text {cod }}
\end{aligned}
$$

As condições adequadas de trabalho são definidas através da análise dos cubos de resposta (Figuras 2 e 3), nos quais os três fatores estudados encontram-se nas arestas em suas formas codificadas, sendo as respectivas respostas, obtidas pelos modelos teóricos estatísticos apresentados nas Equações 4 e 5, colocadas nos vértices dos cubos.

Através das Figuras 2 e 3, verifica-se que o melhor resultado para o fracionamento de óleo de pescado via "winterização" com solvente, tanto no aumento no percentual de ácidos graxos insaturados como na redução no percentual dos saturados, foi obtido no tratamento com o emprego de hexano como solvente (+1), na proporção de $40 \%$ em relação à massa de óleo $(-1)$, e sem o emprego de agitação no $2^{\circ}$ estágio de resfriamento $(-1)$. Sob estas condições, obteve-se um aumento do percentual de aproximadamente 9,2\% no teor de ácidos graxos insaturados e uma redução do percentual de 13,3\% no teor dos saturados.

O melhor solvente utilizado foi o hexano, pois este é mais apolar que a acetona, proporcionando maior solubilização do óleo, aumentando a transferência de calor e a taxa de crescimento dos cristais, ocorrendo maior solubilização dos ácidos graxos poli-insaturados devido à maior apolaridade destes, proporcionando, assim, a concentração dos ácidos graxos saturados em relação à solução, favorecendo sua cristalização.

Em relação à proporção do solvente, sabendo-se que a taxa de crescimento dos cristais é inversamente proporcional à viscosidade da solução, pode-se dizer que, quanto menor a proporção de solvente, mais alta é a viscosidade e mais difícil se torna a troca de matéria entre a fase líquida e a superfície do cristal e mais lento será seu crescimento. O maior enriquecimento dos ácidos graxos insaturados, utilizando a menor proporção de solvente, pode indicar que, nesta condição, ocorre a formação de uma solução estável, em que o crescimento do cristal é favorecido. Ao contrário, alta proporção de solvente pode produzir soluções supersaturadas, sendo a nucleação favorecida, proporcionando a redução da taxa de crescimento dos cristais, diminuindo a eficiência da cristalizalização.

Quanto à agitação no $2^{\circ}$ estágio da “winterização", não é recomendável, pois pode ocorrer a nucleação secundária com a formação de cristais pequenos e difíceis de serem separados.

Resultados semelhantes aos alcançados no presente trabalho, foram observado por López-Martínez et al. (2004) que estudaram a "winterização" via solvente de óleo de semente (Borago officinalis e Echium fastuosum), utilizando acetona, 
Tabela 7. Resultado da análise de variância para as respostas consideradas da matriz experimental estudada.

\begin{tabular}{|c|c|c|c|c|c|c|}
\hline \multirow[t]{2}{*}{ Variável } & \multicolumn{3}{|c|}{ Insaturados } & \multicolumn{3}{|c|}{ Saturados } \\
\hline & Efeito & Teste $F$ & Significância & Efeito & Teste $F$ & Significância \\
\hline (Scod)Solvente & 0,3 & 9,00 & 0,2048 & 0,675 & 29,16 & 0,1166 \\
\hline (PScod)Proporção & $-2,6$ & 676,00 & 0,0245 & $-3,575$ & 817,96 & 0,0223 \\
\hline$(\mathrm{Scod})(\mathrm{PScod})$ & $-1,05$ & 110,25 & 0,0604 & $-1,375$ & 121,00 & 0,0577 \\
\hline$(\mathrm{Scod})($ Acod $)$ & $-3,15$ & 992,25 & 0,0202 & $-4,325$ & 1197,16 & 0,0184 \\
\hline
\end{tabular}

Correlação para a análise de insaturados: $\mathrm{R}=99,95 \%$; e correlação para a análise de saturados $\mathrm{R}=99,96 \%$.

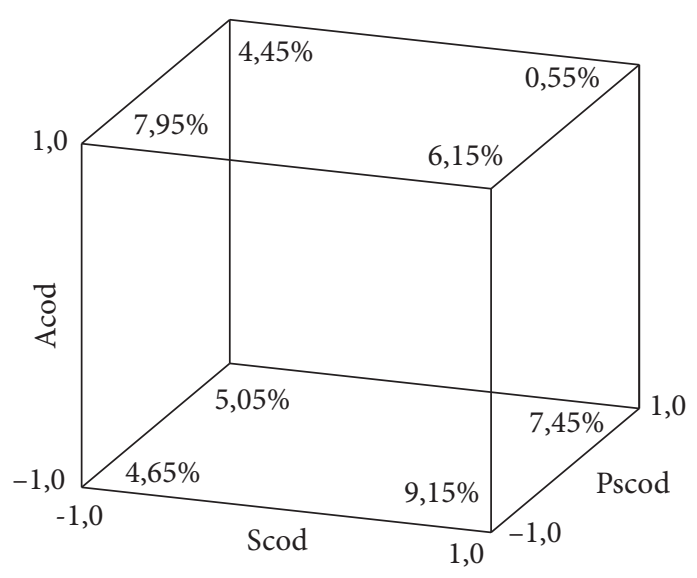

Figura 2. Cubos de resposta para as variações percentuais na "winterização" com solvente do óleo de pescado: percentagem de insaturados.

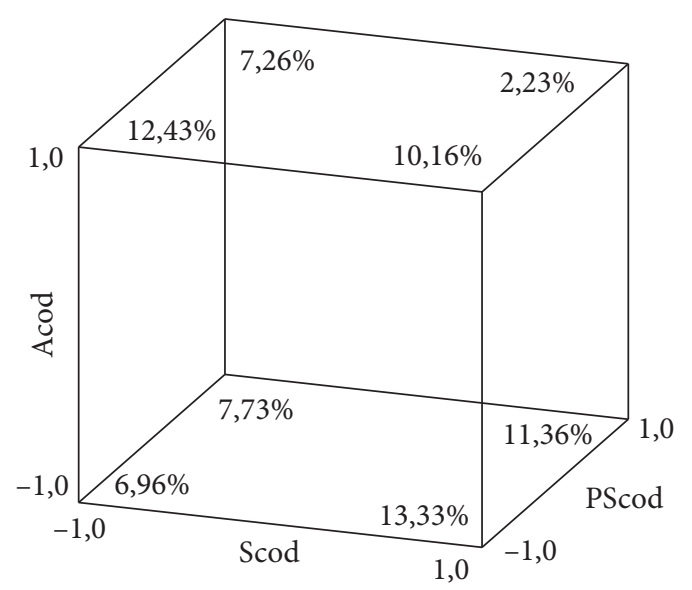

Figura 3. Cubos de resposta para as variações percentuais na "winterização" com solvente do óleo de pescado: percentagem de saturados.

hexano, isobutanol e etanol em diferentes proporções e obtendo melhor resultado com a utilização de hexano como solvente, na menor proporção para todos os solventes utilizados.

\section{Conclusões}

O fracionamento do óleo de pescado via "winterização" com solvente produziu a cristalização dos ácidos graxos saturados e o enriquecimento da concentração dos ácidos insaturados (64,3\% em relação ao óleo).

As condições de trabalho definidas como adequadas foram o emprego de hexano como solvente, na proporção de $40 \%$ em relação à massa de óleo e sem o emprego de agitação durante o $2^{\circ}$ estágio da curva de resfriamento. Sob as condições recomendadas, obteve-se um aumento no percentual de ácidos graxos insaturados de aproximadamente $9,2 \%$ e uma redução no percentual de saturados de $13,3 \%$ em relação ao óleo branqueado.

\section{Agradecimentos}

Os autores agradecem à CAPES (Coordenação de Aperfeiçoamento de Pessoal de Nível Superior) pela concessão da bolsa de estudos.

\section{Referências bibliográficas}

AMERICAN OIL CHEMISTS SOCIETY - AOCS. Official and Tentative Methods of American Oil Chemists, Society. 3 ed. USA, 1980.

AMERICAN OIL CHEMISTS SOCIETY - AOCS. Official Methods and Recommended Pratices of the American Oil Chemist's Society. 5 ed. USA, 2002.

BERNARDINI, E. Tecnologia de Aceites y Grasas. Madrid, Espana: Alhambra S A, 1986.

BOX, G. E. P.; HUNTER, W. G.; HUNTER, J. S. Statistics for Experiments: An Introduction to Design, Data Analysis and Model Building. New York: John Wiley \& Sons, 1978.

COZZOLINO, D. et al. Multivariate determination of free fatty acids and moisture in fish oils by partial leassquare regression and nearinfrared spectroscopy. LWT, Food Science and Technology, v. 38, n. 8, p. 821-828, 2005.

CUNHA, D. C.; SOUZA-SOARES, L. A.; PINTO, L. A. A. Estudo do fracionamento físico do óleo de pescado via "winterização". Brazilian Journal of Food Technology, v. 97, n. 5, p.183-188, 2002.

HOFFMANN, G. The Chemistry and Technology of Edible Oils and Fats and their High Fat Products. London: Academic Press, 1989. 
LIU, S. et al. Concentration of docosahexaenoic acid (DHA) of tuna oil by urea complexation: optimization of process parameters. Journal of Food Engineering, n. 73, p. 203-209, 2006.

LÓPEZ-MARTÍNEZ, J. C; CAMPRA-MADRID, P.; GUIL-GUERRERO, J. L. $\gamma$-Linolenic acid enrichment from borago officinalis and echium fastuosum seed oils and fatty acids by low temperature crystallization. Journal of Bioscience and Bioengineering, v. 97, n. 5, p. 294-298, 2004

MAIA, E. L. Otimização da metodologia para caracterização de constituintes lipídicos e determinação da composição em ácidos graxos e aminoácidos de peixes de água doce. Campinas, 1992. Tese (Doutorado em Ciência de Alimentos), Faculdade de Engenharia de Alimentos, Universidade Estadual de Campinas UNICAMP.

MASTROBATTISTA, G.; GABRIELE, F. Valutazione di una nuova tecnica di raffinazione su oli di sansa. Industrie Alimentari, XXXI (maggio), p. 447-449, 1992.
MORAIS, M. M. et al. Estudo do processo de refino de óleo de pescado. Revista do Instituto Adolfo Lutz, v. 60, n. 1, p. 23-33, 2001.

NAVARRO-GARCÍA, G. et al. Characterization of the lipid composition and natural antioxidants in the liver oil of Dasyatis brevis and Gymnura marmorata rays. Food Chemistry, n. 87, p. 89-96, 2004.

OCKERMAN, H. W.; HANSEN, C. Industrialización de Sub-produtos de Origem Animal. Zaragoza, España: Acribia, 1994.

SIMÕES, I. S.; GIOIELLI, L. A. Cristalização e polimorfismo de gorduras. São Paulo: Ó \& G Alimentos, 1999. p.20-29.

TRUjILlo-QuiJANO, J. A.; ESTEVES, W.; WIRTH, H. G. Considerações técnico-econômicas sobre o fracionamento de óleo-de-dendê. Boletim da Sociedade Brasileira de Ciência e Tecnologia de Alimentos, v.2 2, n. 1, p. 37-52, 1988.

THOMAS III, A. E. Fractionation and Winterization: Processes and Products. In: NORRIS, F. A. (Ed). Bailey's Industrial Oil and Fat Products. 4 ed. USA: John Wiley \& Sons, 1985. p. 1-39.

ZALIHA, O. et al. Crystallization properties of palm oil by dry fractionation. Food Chemistry, n. 86, p. 245-250, 2004. 